Article

\title{
Total Factor Efficiency Study on China's Industrial Coal Input and Wastewater Control with Dual Target Variables
}

\author{
Lingguang $\mathrm{Li}^{1}$ (D) and Weixin Yang ${ }^{2, *(\mathbb{D})}$ \\ 1 School of Mathematical Sciences, Tongji University, Shanghai 200092, China; lilg@tongji.edu.cn \\ 2 Business School, University of Shanghai for Science and Technology, Shanghai 200093, China \\ * Correspondence: iamywx@outlook.com; Tel.: +86-21-5596-0082
}

Received: 27 April 2018; Accepted: 19 June 2018; Published: 21 June 2018

\begin{abstract}
After decades of economic development in China, its energy consumption still relies heavily on coal, and the dominant segment of our economy-the industrial sectors with large coal input-have caused massive wastewater pollution. To provide a decision-making basis for the sustainable development of Chinese industry and economy, this paper selected 39 industrial sectors in China and studied their Total Factor Efficiency (TFE) using Dual Target Variables of coal input and wastewater control during 2003-2014. The results showed that $82.05 \%$ of the total industries within our study scope had an efficiency level below 0.5 . Over the entire study period of 2003-2014, only the national monopoly industries and high and new tech industries were able to maintain the optimal efficiency level. In comparison with the existing Data Envelope Analysis (DEA) Model and studies on TFE using multi-target variables methods, this paper has probably made two improvements. First, we improved the original DEA model to calculate the TFE of two target variables (coal input and wastewater control) at the same time. Second, we developed an algorithm to calculate the TFE of industrial coal input and wastewater control from 2003 to 2014, based on the official input-output statistics of China.
\end{abstract}

Keywords: DEA; dual target variables; industrial coal input; wastewater control; Total Factor Efficiency (TFE)

\section{Introduction}

Although since the reform and opening-up in 1978, China has experienced astonishing economic growth [1], its main energy sources are still limited to coal, oil, and gas; in which coal has taken the biggest proportion in energy consumption, especially since 2000 (see Figure 1 below) [2]. This energy consumption structure above is decided by the resource distribution and energy utilization technology of China. In resource reserve and distribution, China is rich in coal but poor in oil and gas [1]. On the other hand, the utilization and technology related to clean energy and renewable energy has just recently begun in China [3-5]. As shown in Figure 1, the proportion of clean energy and renewable energy in China's energy consumption structure is very small, which will not overtake the dominant position of fossil fuels in the near future [6,7]. Therefore, from 2015-2020 and even many years after, China's economy will remain dependent on coal as the main energy source. 


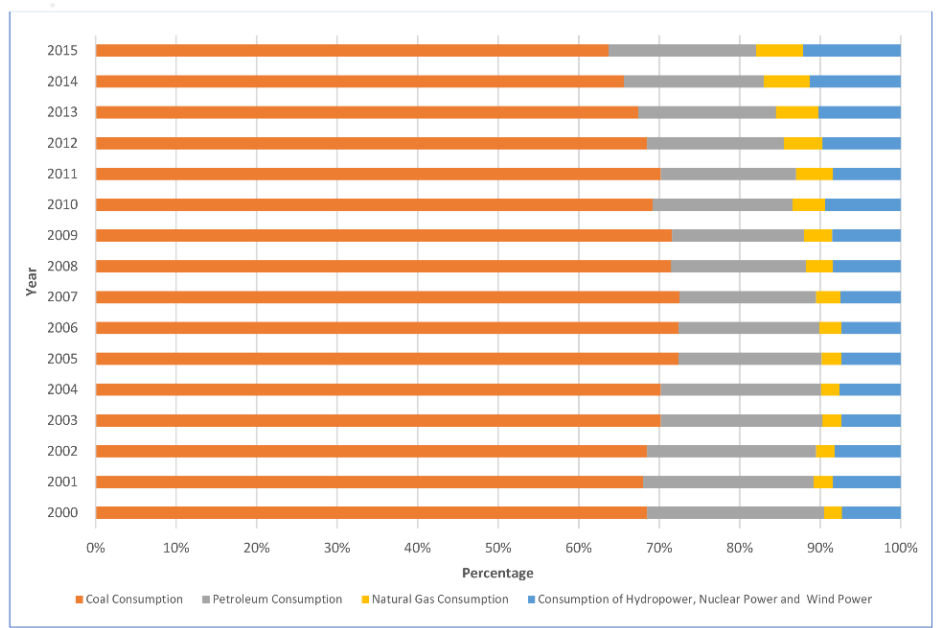

Figure 1. Energy consumption structure in China, 2000-2015 (measurement unit: 10 thousand tons of standard coal.) [2].

As the biggest and most typical industry in China's economy [2], the industrial sector has followed an extensive development path, achieving growth in industrial added value through massive energy input, especially from coal. This growth model has brought about two serious results. First, on the input side, given the limited improvement in energy utilization efficiency, the increasing input of coal and other energy resources has resulted in massive waste of energy. Second, on the output side, with the rapid growth of industrial added value and Gross Domestic Product (GDP), industrial production has also caused serious pollution issues, especially industrial wastewater, which has become a growing threat to the environment and both physical and mental health of the population (see Figure 2 below) [2].

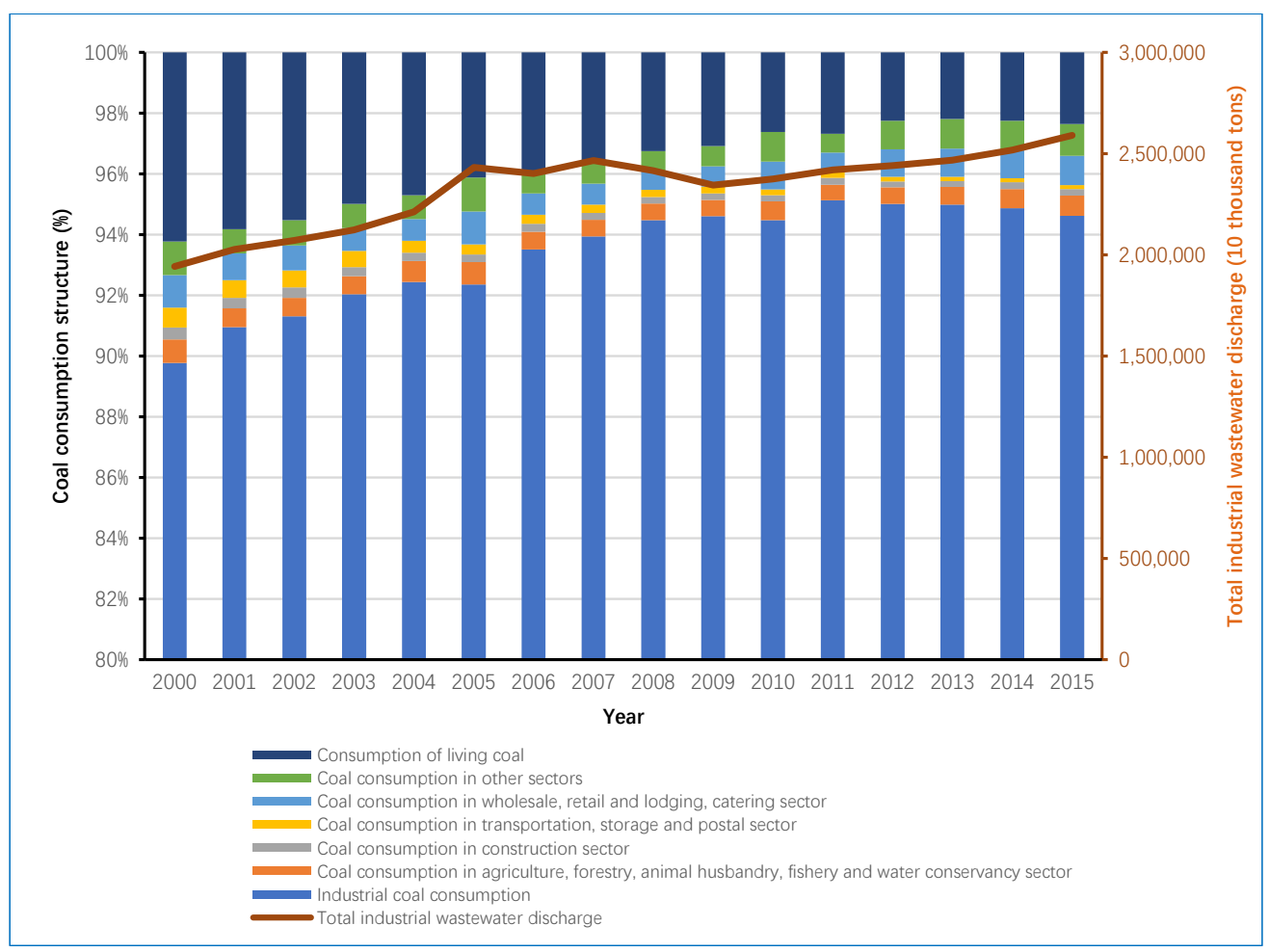

Figure 2. Coal consumption structure and industrial wastewater discharge in China, 2000-2015 [2]. 
In China, the wastewater from industrial coal production is mainly due to the following:

First, at the stage of raw coal mining, large amounts of acid mine drainage is generated due to the oxidation of sulfide contained in coal when exposed to air and water, which dissolves a large number of heavy metal ions and cause great damage to water resources due to its low $\mathrm{pH}$ value [8-10].

Second, at the stage of coal processing and cleaning, the wastewater generated from the wet process or wet techniques contains large numbers of particles with diameter less than $50 \mu \mathrm{m}$ and made of pulverized coal, sand, clay, and siltstone. This kind of wastewater is one of the major pollution sources in the coal industry because it is very stable and cannot use methods of natural sedimentation to separate the particles [11-13].

Last, at the stage of combustion, large amounts of liquid pollutant are emitted. The traditional coal-cleaning techniques in China's coal industry can only get rid of most of the inorganic sulfide in the coal, while the rest enters the atmosphere in the form of $\mathrm{SO}_{2}$ at the stage of combustion and eventually enters and pollutes water resource in the form of acid rain. To make things worse, after absorbing tiny fly ash particulates, the acid rain generates $\mathrm{SO}_{3}$ with higher toxicity under the influence of ferric oxide through catalyzed oxidation. The synergy effect between liquid pollutants and air pollutants causes diseases, including fibrotic lesion of the lung and emphysema, resulting in huge health damage and economic loss [14-16].

Integrated wastewater governance has become one of the key research areas in industrial wastewater management $[17,18]$. When evaluating wastewater control, researchers are putting more emphasis on comprehensive wastewater management, balancing both cost and effective supervision. Various new techniques including recovering and recycling, treatment technologies, and efficient operations have been recognized by the academia [19-21].

In recent studies, the multiple objective optimization model has been widely developed and used [22-24]. However, in terms of the Chinese industry, there are a few studies that focus on multiple target efficiencies by connecting the input and the output within the DEA model.

Therefore, the main contributions of this paper lie in:

(1) Considering the major input and output variables of China's industry, this paper simultaneously calculated the TFE of coal input and wastewater output in China's industrial sectors, which provided the decision-making basis for sustainable development.

(2) Through improvement of the DEA model and development of an algorithm, this paper realized calculation of TFE of the input and output variables at the same time, enriching theoretical and computational literature of the model.

In the following parts of this paper, we improved upon the traditional DEA Model in Section 2, by putting total industrial coal consumption and industrial wastewater emission of China, respectively, on the input and output side of the DEA Model and constructing a theoretical model that can calculate the Dual Target Variable TFE of both the input factor and undesirable output factor. The data source of the input and output variables was the State Statistical Bureau of China. In Section 3, we utilized the MATLAB algorithm for Dual Target Variable TFE developed by ourselves to calculate the TFE of coal input and industrial wastewater control in 39 Chinese industries. We further analyzed the difference in TFE of different industries as well as the reasons for fluctuation in Section 4. Finally, Section 5 served as the summary of this paper and provided related policy recommendations.

\section{Methodology and Data}

\subsection{DEA Model with Dual Target Variable}

This paper adopted an improved DEA Model to measure the Dual Target Variable TFE of coal input and wastewater control of 39 industries in China. Based on existing studies using the DEA Model for wastewater control efficiency assessment, we selected 39 industries as Decision Making Units $\left(D M U_{i}, 1 \leq i \leq 39\right)$ adopted labor, capital, and coal input as input factors of the production 
process; chose industrial added value, industrial wastewater and waste gas emission, and industrial solid waste emission as output factors; and calculated the optimal Total Factor Efficiency (TFE) of coal input and wastewater control on the production possibility frontier with the help of the DEA Model. The optimal TFE above is based on the assumption of maximum industrial output (calculated by the Output Method) of each DMU $\left(D M U_{i}\right)$.

The input and output variables involved in the model are listed as follows:

I. Input variables

(1) Labor. We measured the labor input of each DMU by the average number of employees in those 39 industries from 2003 to 2014. The data source was the "China Statistical Yearbook (2004-2015)", published by the National Bureau of Statistics [25].

(2) Capital: We calculated capital input of different industries from 2003 to 2014 based on official statistics from the "China Statistical Yearbook (2004-2015)" [25]. The calculation is more complicated than that of labor. Here, we defined capital input as the stock fixed assets investment of the 39 industries, and adopted the "Perpetual Inventory" accounting method to estimate the Capital Stock from annual fixed assets investment based on the common practice of similar studies:

$$
K_{n, t}=K_{n, t-1}+I_{n, t}-D_{n, t}=\left(1-d_{n}\right) K_{n, t-1}+I_{n, t}
$$

In Equation (9) above, $K_{n, t}$ is the capital stock from fixed assets investment of industry $n$ in year $t$, while $K_{n, t-1}$ is the capital stock of last year (year $t-1$ ) by industry $n$. $I_{n, t}$ represents the new fixed assets investment in year $t$ by industry $n ; D_{n, t}$ is the fixed assets depreciation of industry $n$ in year $t$; while $d_{n}$ stands for the fixed assets depreciation rate of that industry. Therefore, the capital stock from fixed assets investment of each industry in the previous year (year $t-1$ ) can be calculated by [26]:

$$
K_{n, t-1}=I_{n, t-1} /\left(k_{n}+d_{n}\right)
$$

In Equation (10) above, $I_{n, t-1}$ is the new fixed assets investment in the last year of that industry; $k_{n}$ is the growth rate of fixed assets investment in that industry. To align with the common practice in similar studies, we used the geometric average to represent the average growth rate of fixed assets investment $k_{n}$ in an $m$-year period:

$$
k_{n}=\left(I_{n, m} / I_{n, 0}\right)^{1 / m}-1
$$

From Equations (9)-(11), we can obtain the average capital stock of each industry in each year. In order to ensure the comparability and accuracy of our results, we set 2003 as the base year and adjusted the capital stock of the following years by using the Fixed Asset Investment Price Index.

(3) Coal input: We adopted the "Coal Consumption (10 thousand tons)" data of each industry in each year from the "China Statistical Yearbook (2004-2015)" and "China Energy Statistical Yearbook (2004-2015)", published by the National Bureau of Statistics of China [25,27], as our Coal Input Variable.

II. Output variables

(1) Industrial added value: This is a straightforward output measurement of each industry, and a direct component of our country's GDP, calculated by the Output Method. Throughout the years, the industrial added value has taken the largest proportion in our country's annual GDP [1]. Therefore, the industrial added value is not only the most important indicator of a certain industry's output level, but also an important indicator for China's economic 
growth. Our data came from the "China Statistical Yearbook (2004-2015)" published by the National Bureau of Statistics of China [25], and we further adjusted the annual industrial added value of each industry by use of the GDP Deflator with 2003 being the base year.

(2) Industrial wastewater emission, waste gas emission, and solid waste emission as three Undesirable Outputs. It is worth pointing out that although this study focused on the efficiency of industrial wastewater control, in practice, industrial waste gas and solid waste are also inevitable products of industrial production worldwide, especially for production processes that involve coal as an input. As we all know, the calculation result of the DEA Model is impacted by the choice of Input-Output variables. If we exclude these two undesirable outputs, it would not comply with the actual industrial production process and would cause inaccurate DEA calculation result due to neglect of major output variables. Therefore, we decided to include waste gas and solid waste into our model as outputs to reflect actual industrial production. It is also worth mentioning that many researchers put undesirable outputs on the input side of the DEA Model [28-30]. Although this practice has its rationality, given the setting of the DEA Model, undesirable output itself is still an output; it is the product of various input factors. Therefore, we decided it is inappropriate and contradictory to treat undesirable outputs as inputs for the purpose of assessing the Dual Target Variable TFE of coal input and industrial wastewater control (as an output) at the same time. Based on the theoretical model in Section 2.1 and with help of our MATLAB algorithm, we were able to simultaneously calculate the TFE of coal input and undesirable output (industrial wastewater) within the DEA framework by putting undesirable variables on the output side. Our calculation was based on wastewater, waste gas and solid waste emission data of various industries officially published in the "China Statistical Yearbook (2004-2015)" by the National Bureau of Statistics of China [25].

First, the TFE of industrial coal input TFEC $C_{i, t}$ and TFE of wastewater control TFEPW $W_{i, t}$ can be written as:

$$
\begin{gathered}
\text { TFEC }_{i, t}=\frac{c_{i, t}}{C_{i, t}} \\
\text { TFEPW }_{i, t}=\frac{w_{i, t}}{W_{i, t}}
\end{gathered}
$$

In the equation above, $c_{i, t}$ presents the expected coal input on the production possibility frontier of $D M U_{i}$, while $C_{i, t}$ is the actual coal input of $D M U_{i}$ at period $t . w_{i, t}$ is the expected wastewater emission on the production possibility frontier of $D M U_{i}$, while $W_{i, t}$ is the actual wastewater emission of $D M U_{i}$ at period $t$.

After defining the TFE of coal input $\left(T F E C_{i, t}\right)$ and wastewater control $\left(T F E P W_{i, t}\right)$, let us introduce the improved DEA-SBM Model. In our model, there are $s$ Decision Making Units $\left(D M U_{i}, 1 \leq i \leq s\right)$. For each $D M U_{i}$, the input vector of $m$ production factors at period $t$ is $x_{i}$; its desirable output vector of $n_{g}$ products is written as $y_{i}^{g}$, and its undesirable output vector of $n_{b}$ products is expressed as $y_{i}^{b}$. Let the Input Matrix $X$, Undesirable Output Matrix $Y^{b}$ and Desirable Output Matrix $Y^{g}$ be:

$$
\begin{gathered}
X=\left(x_{j i}\right) \in R_{m \times s} \\
Y^{b}=\left(y_{j i}^{b}\right) \in R_{n_{b} \times s} \\
Y^{g}=\left(y_{j i}^{g}\right) \in R_{n_{g} \times s}
\end{gathered}
$$

In Formulas (3)-(5) above, $x_{i}=\left(x_{1 i}, x_{2 i}, \ldots, x_{m, i}\right)^{t} ; y_{i}^{b}=\left(y_{1 i}^{b}, y_{2 i}^{b}, \ldots, y_{n_{g} i}^{b}\right)^{t} ; y_{i}^{g}=\left(y_{1 i}^{g}, y_{2 i}^{g}, \ldots, y_{n_{g} i}^{g}\right)^{t}$, which respectively represent the $i^{\text {th }}$ column vector of Input Matrix $X$, Undesirable Output Matrix $Y^{b}$ and Desirable Output Matrix $Y^{g}$, i.e., the $i^{\text {th }}$ Decision Making Unit $\left(\mathrm{DMU}_{\mathrm{i}}\right)^{\prime} \mathrm{s}$ actual input vector, actual undesirable output vector and actual desirable output vector. When $x_{i}, y_{i}^{b}$ and $y_{i}^{g}$ are all larger than 
zero, it means the actual input is higher than the optimal input on the production possibility frontier (i.e., $x \geq X \lambda$ ). It also means that the actual undesirable output is higher compared with the undesirable output on the production possibility frontier (i.e., $y^{b} \geq Y^{b} \lambda$ ), and that the actual desirable output is lower than the optimal desirable output level on the production possibility frontier (i.e., $y^{g} \leq \gamma^{g} \lambda$ ). $\lambda$ stands for the ratio vector between the actual output compared with the optimal output on the production possibility frontier. Therefore, the Input-Output possibility set $\left(p_{i}\right)$ of each $D M U_{i}$ could be written as:

$$
p_{i}=\left\{\left(x, y^{b}, y^{g}\right) \mid x \geq X \lambda, y^{b} \geq Y^{b} \lambda, y^{g} \leq Y^{g} \lambda, \lambda \geq 0\right\}
$$

The efficiency index $\rho_{i}$ of the $i^{\text {th }}$ Decision Making Unit $\left(D M U_{i}\right)$ can be defined as below:

$$
\rho_{i}=\frac{\frac{1}{m}\left(\sum_{j=1}^{m} \frac{\sum_{r=1}^{s} x_{j, r}^{g} \lambda_{r}}{x_{j, i}^{g}}\right)}{\frac{1}{n_{g}+n_{b}}\left(\sum_{j=1}^{n_{g}} \frac{\sum_{r=1}^{s} y_{j, r}^{g} \lambda_{r}}{y_{j, i}^{g}}+\sum_{j=1}^{n_{b}} \frac{y_{j, i}^{b}}{\sum_{r=1}^{s} y_{j, r}^{b} \lambda_{r}}\right)}
$$

By definition, the efficiency indicator $\rho_{i}$ should range from 0 to 1 , and monotonically decrease with the increase in "Input Slack" and "Output Slack". The increase in "Input Slack" means there is waste in input factors, so the efficiency is lower. The increase in "Undesirable Output Slack" means the actual undesirable output is getting farther away from the undesirable output level on the production possibility frontier, so the efficiency is also lower. The increase in "Desirable Output Slack" means the actual output is moving away from the optimal desirable output level on the production possibility frontier, and therefore the production efficiency is also lower. When $\rho=1$, which means the "Input Slack", "Undesirable Output Slack", and "Desirable Output Slack" are all 0, the input-output of $D M U_{i}$ has reached optimization and the highest production efficiency.

Based on the discussion above, we established our improved DEA-SBM Model as below:

$$
\begin{gathered}
\min \rho_{i}=\frac{\frac{1}{m}\left(\sum_{j=1}^{m} \frac{\sum_{r=1}^{s} x_{j, r}^{g} \lambda_{r}}{x_{j, i}^{g}}\right)}{\frac{1}{n_{g}+n_{b}}\left(\sum_{j=1}^{n_{g}} \frac{\sum_{r=1}^{s} y_{j, r}^{g} \lambda_{r}}{y_{j, i}^{g}}+\sum_{j=1}^{n_{b}} \frac{y_{j, i}^{b}}{\sum_{r=1}^{s} y_{j, r}^{b} \lambda_{r}}\right)} \\
\text { s.t. } \\
X^{g} \lambda \leq x^{g} \\
Y^{b} \lambda \leq y^{b}, \\
-Y^{g} \lambda \leq-y^{g}, \\
\lambda \geq 0
\end{gathered}
$$

This paper adopted the fmincon() Function in MATLAB software (The MathWorks, Inc. Natick, MA, USA) widely used for optimization problems of multi-variable constrained nonlinear functions to solve our DEA-SBM Model, and made a few adjustments on the form of the target functions in our DEA-SBM Model. By using matrices of known variables and ratio vectors to express the Slack Variable Vector, we were able to keep our MATLAB algorithm in consistence with the form of the target functions [31-33]. Through multiple tests, the results from this model were highly robust with different initial variable values, and the calculation also showed good convergence and high accuracy. Therefore, we have a good reason to believe that the calculation result from this model is the global optimal solution rather than the local optimal solution of our optimization problem [34-36].

When calculating the Total Factor Efficiency (TFE) of industrial wastewater control with our DEA-SBM Model, we selected three input variables $X$ (labor, capital, and coal input), one desirable output variable $Y^{g}$ (industrial added value), and three undesirable output variables $Y^{b}$ (industrial wastewater emission, industrial waste gas emission, and industrial solid waste emission). 


\subsection{Industrial Classification in China}

Based on the Industry Classification Guideline (GB/T4754-2002 and GB/T4754-2011) issued by the National Bureau of Statistics of China [2], we have selected 39 industries as well as input and output variables as below, based on our improved DEA Model (see Table 1 below):

Table 1. The industrial classification in China [2].

\begin{tabular}{|c|c|}
\hline No. & Industry \\
\hline 1 & Coal mining and washing \\
\hline 2 & Oil and gas extraction \\
\hline 3 & Black metal mining \\
\hline 4 & Nonferrous metal mining \\
\hline 5 & Nonmetallic mining \\
\hline 6 & Other mining \\
\hline 7 & Sideline food processing \\
\hline 8 & Food manufacturing \\
\hline 9 & Beverage manufacturing \\
\hline 10 & Tobacco products \\
\hline 11 & Textile \\
\hline 12 & Textile and garment, shoes, hats manufacturing \\
\hline 13 & Leather, fur, feather (velvet) and its products \\
\hline 14 & Wood processing and wood, bamboo, rattan, brown, grass products \\
\hline 15 & Furniture manufacturing \\
\hline 16 & Paper and paper products \\
\hline 17 & Printing and recording media \\
\hline 18 & Cultural and educational sporting goods manufacturing \\
\hline 19 & Oil processing, coking and nuclear fuel processing \\
\hline 20 & Chemical raw materials and chemical products manufacturing \\
\hline 21 & Pharmaceutical manufacturing \\
\hline 22 & Chemical fiber manufacturing \\
\hline 23 & Rubber products \\
\hline 24 & Plastic products \\
\hline 25 & Nonmetallic mineral products \\
\hline 26 & Ferrous metal smelting and rolling processing \\
\hline 27 & Nonferrous metal smelting and rolling processing \\
\hline 28 & Metal products \\
\hline 29 & General equipment manufacturing \\
\hline 30 & Special equipment manufacturing \\
\hline 31 & Transportation equipment manufacturing \\
\hline 32 & Electrical machinery and equipment manufacturing \\
\hline 33 & Communications equipment, computers and other electronic equipment manufacturing \\
\hline 34 & Instrumentation and culture, office machinery manufacturing \\
\hline 35 & Handicrafts and other manufacturing \\
\hline 36 & Waste resources and waste materials recycling \\
\hline 37 & Electricity, heat production and supply \\
\hline 38 & Gas production and supply \\
\hline 39 & Water production and supply \\
\hline
\end{tabular}




\subsection{MATLAB Algorithm}

We developed our own MATLAB algorithm (please see Appendix A) based on existing research and the improved DEA Model in Section 2.1. Our algorithm simultaneously calculates the TFE of both coal input and industrial wastewater control by putting the former on the input and putting the latter on the output side, enabling calculation and assessment of Dual Target Variable TFE covering both the input (coal) and the output (industrial wastewater) within the DEA Model.

\section{Results}

As shown in Section 2.1, there are three kinds of variables in the improved DEA-SBM model of this paper: (1) Three input variables-labor input, capital investment, and total coal consumption; (2) three undesirable output variables—industrial wastewater emission, industrial waste gas emission, and industrial solid waste emission; and (3) one desirable output variable-industrial added value.

Based on the improved DEA-SBM Model and the input-output variables listed in Section 2.1, this paper brought the values of the above variables into the improved DEA-SBM model, and solved the optimization problem of the constrained multi-variable nonlinear functions with the "fmincon()" function in MATLAB software. Therefore, the theoretical value of each variable can be calculated. We finally obtained the TFE value of coal and wastewater through comparison between the theoretical value and their actual values (as shown in Tables $2-4$ below). 
Table 2. TFE of coal input and wastewater control in different industries of China, 2003-2006.

\begin{tabular}{|c|c|c|c|c|c|c|c|c|c|}
\hline \multirow{3}{*}{ No. } & \multirow{3}{*}{ Industry } & \multicolumn{8}{|c|}{ Year } \\
\hline & & \multicolumn{2}{|c|}{2003} & \multicolumn{2}{|c|}{2004} & \multicolumn{2}{|c|}{2005} & \multicolumn{2}{|c|}{2006} \\
\hline & & Coal & Waste-Water & Coal & Waste-Water & Coal & Waste-Water & Coal & Waste-Water \\
\hline 1 & Coal mining and washing & 0.0370 & 0.3717 & 0.2159 & 0.0213 & 0.0208 & 0.1894 & 0.0266 & 0.1946 \\
\hline 2 & Oil and gas extraction & 1.0000 & 1.0000 & 0.5804 & 0.6147 & 1.0000 & 1.0000 & 1.0000 & 1.0000 \\
\hline 3 & Black metal mining & 0.1215 & 0.0440 & 0.0333 & 0.1393 & 0.2408 & 0.0716 & 0.2436 & 0.0477 \\
\hline 4 & Nonferrous metal mining & 0.0718 & 0.0334 & 0.0505 & 0.1698 & 0.3013 & 0.0329 & 0.3436 & 0.0192 \\
\hline 5 & Nonmetallic mining & 0.1201 & 0.5106 & 0.0337 & 0.0301 & 0.0388 & 0.0592 & 0.0400 & 0.0578 \\
\hline 6 & Other mining & 0.0610 & 0.3663 & 1.0000 & 1.0000 & 0.1354 & 0.0169 & 1.0000 & 1.0000 \\
\hline 7 & Sideline food processing & 0.2182 & 0.0522 & 0.1358 & 0.1060 & 0.1296 & 0.0315 & 0.1412 & 0.0442 \\
\hline 8 & Food manufacturing & 0.1093 & 0.0603 & 0.4419 & 0.2382 & 0.0788 & 0.0372 & 0.0842 & 0.0407 \\
\hline 9 & Beverage manufacturing & 0.0356 & 0.0877 & 0.2984 & 0.1720 & 0.0911 & 0.0366 & 0.0981 & 0.0307 \\
\hline 10 & Tobacco products & 1.0000 & 1.0000 & 1.0000 & 1.0000 & 1.0000 & 1.0000 & 1.0000 & 1.0000 \\
\hline 11 & Textile & 0.0296 & 0.0514 & 0.2511 & 0.1585 & 0.0835 & 0.0257 & 0.0833 & 0.0240 \\
\hline 12 & Textile and garment, shoes, hats manufacturing & 1.0000 & 1.0000 & 0.9883 & 0.4360 & 0.7435 & 0.3713 & 0.4158 & 0.1604 \\
\hline 13 & Leather, fur, feather (velvet) and its products & 0.7009 & 0.1505 & 0.5354 & 0.5631 & 0.5896 & 0.0702 & 0.6231 & 0.0691 \\
\hline 14 & Wood processing and wood, bamboo, rattan, brown, grass products & 0.0259 & 0.1471 & 0.0925 & 0.0606 & 0.0769 & 0.1062 & 0.0939 & 0.2451 \\
\hline 15 & Furniture manufacturing & 1.0000 & 1.0000 & 0.2765 & 0.5574 & 0.7719 & 0.6585 & 0.6314 & 0.9229 \\
\hline 16 & Paper and paper products & 0.1501 & 0.0095 & 0.0436 & 0.0304 & 0.0220 & 0.0043 & 0.0307 & 0.0061 \\
\hline 17 & Printing and recording media & 1.0000 & 1.0000 & 1.0000 & 1.0000 & 0.5176 & 0.6307 & 0.4578 & 0.8300 \\
\hline 18 & Cultural and educational sporting goods manufacturing & 1.0000 & 1.0000 & 0.7850 & 1.0000 & 1.0000 & 1.0000 & 1.0000 & 1.0000 \\
\hline 19 & Oil processing, coking and nuclear fuel processing & 0.0485 & 0.1072 & 0.0251 & 0.0079 & 0.0070 & 0.0426 & 0.0059 & 0.0394 \\
\hline 20 & Chemical raw materials and chemical products manufacturing & 0.0998 & 0.0330 & 0.0523 & 0.0391 & 0.0635 & 0.0550 & 0.0390 & 0.0348 \\
\hline 21 & Pharmaceutical manufacturing & 0.1441 & 0.0854 & 0.1756 & 0.1579 & 0.1464 & 0.0521 & 0.1453 & 0.0503 \\
\hline 22 & Chemical fiber manufacturing & 0.0689 & 0.0193 & 0.0222 & 0.0358 & 0.0396 & 0.0138 & 0.0430 & 0.0147 \\
\hline 23 & Rubber products & 0.0470 & 0.1922 & 0.1231 & 0.0856 & 0.0882 & 0.1327 & 0.0922 & 0.1440 \\
\hline 24 & Plastic products & 0.3843 & 1.0000 & 0.4680 & 0.2716 & 1.0000 & 1.0000 & 0.3332 & 0.5913 \\
\hline 25 & Nonmetallic mineral products & 0.0139 & 0.1048 & 0.0131 & 0.0202 & 0.0198 & 0.2147 & 0.0237 & 0.3181 \\
\hline 26 & Ferrous metal smelting and rolling processing & 0.0199 & 0.0449 & 0.0205 & 0.0332 & 0.0315 & 0.0950 & 0.0222 & 0.0704 \\
\hline 27 & Nonferrous metal smelting and rolling processing & 0.0223 & 0.1011 & 0.0151 & 0.0391 & 0.0512 & 0.0786 & 0.0694 & 0.1173 \\
\hline 28 & Metal products & 0.2624 & 0.2003 & 0.3803 & 0.2923 & 0.3295 & 0.1097 & 0.3838 & 0.1188 \\
\hline 29 & General equipment manufacturing & 0.0983 & 0.4481 & 0.4235 & 0.3881 & 0.4734 & 0.2585 & 0.5076 & 0.3624 \\
\hline 30 & Special equipment manufacturing & 0.0548 & 0.2735 & 0.2143 & 0.1846 & 0.1994 & 0.2025 & 0.2336 & 0.2411 \\
\hline 31 & Transportation equipment manufacturing & 0.0900 & 0.2694 & 0.2534 & 0.3103 & 0.2912 & 0.2124 & 0.3248 & 0.2298 \\
\hline 32 & Electrical machinery and equipment manufacturing & 0.2584 & 0.7502 & 1.0000 & 1.0000 & 1.0000 & 1.0000 & 1.0000 & 1.0000 \\
\hline 33 & $\begin{array}{c}\text { Communications equipment, computers and other electronic } \\
\text { equipment manufacturing }\end{array}$ & 1.0000 & 1.0000 & 1.0000 & 1.0000 & 1.0000 & 1.0000 & 1.0000 & 1.0000 \\
\hline 34 & Instrumentation and culture, office machinery manufacturing & 0.4070 & 0.1824 & 0.1334 & 1.0000 & 0.9095 & 0.3252 & 0.9426 & 0.4014 \\
\hline 35 & Handicrafts and other manufacturing & 1.0000 & 1.0000 & 0.4892 & 0.0566 & 1.0000 & 1.0000 & 0.0605 & 0.4565 \\
\hline 36 & Waste resources and waste materials recycling & 0.0118 & 0.0009 & 0.0677 & 0.1487 & 0.5560 & 0.3478 & 0.7609 & 0.2633 \\
\hline 37 & Electricity, heat production and supply & 0.0096 & 0.0676 & 0.0109 & 0.0052 & 0.0077 & 0.0769 & 0.0048 & 0.0610 \\
\hline 38 & Gas production and supply & 0.0091 & 0.0630 & 0.0521 & 0.0123 & 0.0068 & 0.0452 & 0.0078 & 0.0703 \\
\hline 39 & Water production and supply & 1.0000 & 1.0000 & 0.9424 & 0.5049 & 0.4510 & 0.0192 & 0.4605 & 0.0191 \\
\hline
\end{tabular}


Table 3. TFE of coal input and wastewater control in different industries of China, 2007-2010.

\begin{tabular}{|c|c|c|c|c|c|c|c|c|c|}
\hline \multirow{3}{*}{ No. } & \multirow{3}{*}{ Industry } & \multicolumn{8}{|c|}{ Year } \\
\hline & & \multicolumn{2}{|c|}{2007} & \multicolumn{2}{|c|}{2008} & \multicolumn{2}{|c|}{2009} & \multicolumn{2}{|c|}{2010} \\
\hline & & Coal & Waste-Water & Coal & Waste-Water & Coal & Waste-Water & Coal & Waste-Water \\
\hline 1 & Coal mining and washing & 0.0122 & 0.0697 & 0.0119 & 0.0957 & 0.0201 & 0.1613 & 0.0184 & 0.1241 \\
\hline 2 & Oil and gas extraction & 0.6958 & 0.6657 & 0.7008 & 0.5751 & 0.3538 & 0.7053 & 0.3143 & 0.5112 \\
\hline 3 & Black metal mining & 0.2653 & 0.0604 & 0.1728 & 0.0583 & 0.2063 & 0.0830 & 0.1598 & 0.0834 \\
\hline 4 & Nonferrous metal mining & 0.3734 & 0.0232 & 0.3334 & 0.0230 & 0.3256 & 0.0302 & 0.2846 & 0.0247 \\
\hline 5 & Nonmetallic mining & 0.0317 & 0.0589 & 0.0345 & 0.0709 & 0.0240 & 0.0749 & 0.0255 & 0.0672 \\
\hline 6 & Other mining & 1.0000 & 1.0000 & 1.0000 & 1.0000 & 1.0000 & 1.0000 & 0.0983 & 0.0229 \\
\hline 7 & Sideline food processing & 0.1267 & 0.0308 & 0.0920 & 0.0294 & 0.0848 & 0.0360 & 0.0749 & 0.0297 \\
\hline 8 & Food manufacturing & 0.0751 & 0.0428 & 0.0566 & 0.0390 & 0.0715 & 0.0476 & 0.1075 & 0.0624 \\
\hline 9 & Beverage manufacturing & 0.0927 & 0.0294 & 0.3062 & 0.0979 & 0.1891 & 0.0861 & 0.1692 & 0.0467 \\
\hline 10 & Tobacco products & 1.0000 & 1.0000 & 1.0000 & 1.0000 & 1.0000 & 1.0000 & 1.0000 & 1.0000 \\
\hline 11 & Textile & 0.0740 & 0.0218 & 0.0634 & 0.0214 & 0.0619 & 0.0230 & 0.0578 & 0.0196 \\
\hline 12 & Textile and garment, shoes, hats manufacturing & 0.3621 & 0.1539 & 0.3209 & 0.1498 & 0.3156 & 0.1714 & 0.2663 & 0.1726 \\
\hline 13 & Leather, fur, feather (velvet) and its products & 0.5919 & 0.0626 & 0.5625 & 0.0567 & 0.5278 & 0.0661 & 0.5406 & 0.0481 \\
\hline 14 & Wood processing and wood, bamboo, rattan, brown, grass products & 0.0962 & 0.2102 & 0.0763 & 0.2216 & 0.0713 & 0.1871 & 0.0654 & 0.1874 \\
\hline 15 & Furniture manufacturing & 0.8279 & 0.3445 & 0.6288 & 0.3542 & 0.6646 & 0.3884 & 0.5250 & 0.2760 \\
\hline 16 & Paper and paper products & 0.0573 & 0.0097 & 0.0254 & 0.0060 & 0.0232 & 0.0067 & 0.0247 & 0.0059 \\
\hline 17 & Printing and recording media & 0.5961 & 0.3782 & 0.5317 & 0.4623 & 0.5358 & 0.4324 & 0.4246 & 0.4015 \\
\hline 18 & Cultural and educational sporting goods manufacturing & 1.0000 & 1.0000 & 1.0000 & 1.0000 & 1.0000 & 1.0000 & 1.0000 & 1.0000 \\
\hline 19 & Oil processing, coking and nuclear fuel processing & 0.0074 & 0.0604 & 0.0061 & 0.0726 & 0.0101 & 0.1180 & 0.0224 & 0.1891 \\
\hline 20 & Chemical raw materials and chemical products manufacturing & 0.0372 & 0.0396 & 0.0294 & 0.0431 & 0.0308 & 0.0477 & 0.0294 & 0.0394 \\
\hline 21 & Pharmaceutical manufacturing & 0.1385 & 0.0525 & 0.1037 & 0.0477 & 0.1013 & 0.0487 & 0.0886 & 0.0401 \\
\hline 22 & Chemical fiber manufacturing & 0.1058 & 0.0416 & 0.1050 & 0.0391 & 0.0648 & 0.0372 & 0.1228 & 0.0408 \\
\hline 23 & Rubber products & 0.0892 & 0.1467 & 0.0681 & 0.1488 & 0.0646 & 0.1576 & 0.0780 & 0.1811 \\
\hline 24 & Plastic products & 0.3177 & 0.5072 & 0.2225 & 0.4411 & 0.1778 & 0.5429 & 0.1555 & 0.3953 \\
\hline 25 & Nonmetallic mineral products & 0.0209 & 0.3018 & 0.0173 & 0.3693 & 0.0171 & 0.4227 & 0.0171 & 0.3862 \\
\hline 26 & Ferrous metal smelting and rolling processing & 0.0306 & 0.1170 & 0.0220 & 0.1167 & 0.0241 & 0.1584 & 0.0425 & 0.2811 \\
\hline 27 & Nonferrous metal smelting and rolling processing & 0.0652 & 0.1386 & 0.0443 & 0.1491 & 0.0501 & 0.1921 & 0.0258 & 0.1564 \\
\hline 28 & Metal products & 0.3838 & 0.0889 & 0.2859 & 0.1065 & 0.2748 & 0.1070 & 0.2615 & 0.0915 \\
\hline 29 & General equipment manufacturing & 0.5180 & 0.4127 & 0.3807 & 0.3572 & 0.3676 & 0.4241 & 0.3237 & 0.3583 \\
\hline 30 & Special equipment manufacturing & 0.2282 & 0.3205 & 0.1821 & 0.2916 & 0.1629 & 0.3113 & 0.1344 & 0.2901 \\
\hline 31 & Transportation equipment manufacturing & 0.3340 & 0.3114 & 0.2719 & 0.2448 & 0.2524 & 0.2839 & 0.2240 & 0.2436 \\
\hline 32 & Electrical machinery and equipment manufacturing & 1.0000 & 1.0000 & 1.0000 & 1.0000 & 1.0000 & 1.0000 & 1.0000 & 1.0000 \\
\hline 33 & $\begin{array}{c}\text { Communications equipment, computers and other electronic } \\
\text { equipment manufacturing }\end{array}$ & 1.0000 & 1.0000 & 1.0000 & 1.0000 & 1.0000 & 1.0000 & 1.0000 & 1.0000 \\
\hline 34 & Instrumentation and culture, office machinery manufacturing & 1.0000 & 1.0000 & 1.0000 & 1.0000 & 1.0000 & 1.0000 & 1.0000 & 1.0000 \\
\hline 35 & Handicrafts and other manufacturing & 0.0750 & 0.2399 & 0.0595 & 0.4879 & 0.0613 & 0.2851 & 0.0534 & 0.3283 \\
\hline 36 & Waste resources and waste materials recycling & 0.9494 & 0.1662 & 0.5540 & 0.2506 & 0.4781 & 0.1883 & 0.3436 & 0.1295 \\
\hline 37 & Electricity, heat production and supply & 0.0041 & 0.0942 & 0.0033 & 0.0774 & 0.0061 & 0.1755 & 0.0058 & 0.1914 \\
\hline 38 & Gas production and supply & 0.0085 & 0.1064 & 0.0099 & 0.1329 & 0.0077 & 0.1730 & 0.0064 & 0.1483 \\
\hline 39 & Water production and supply & 0.2649 & 0.0467 & 0.3156 & 0.0204 & 0.5187 & 0.0199 & 0.1509 & 0.0108 \\
\hline
\end{tabular}


Table 4. TFE of coal input and wastewater control in different industries of China, 2011-2014.

\begin{tabular}{|c|c|c|c|c|c|c|c|c|c|}
\hline \multirow{3}{*}{ No. } & \multirow{3}{*}{ Industry } & \multicolumn{8}{|c|}{ Year } \\
\hline & & \multicolumn{2}{|c|}{2011} & \multicolumn{2}{|c|}{2012} & \multicolumn{2}{|c|}{2013} & \multicolumn{2}{|c|}{2014} \\
\hline & & Coal & Waste-Water & Coal & Waste-Water & Coal & Waste-Water & Coal & Waste-Water \\
\hline 1 & Coal mining and washing & 0.0189 & 0.2541 & 0.0160 & 0.2545 & 0.0152 & 0.2501 & 0.0158 & 0.2595 \\
\hline 2 & Oil and gas extraction & 0.4309 & 0.5271 & 0.2913 & 0.5270 & 0.2870 & 0.5270 & 0.5903 & 0.5142 \\
\hline 3 & Black metal mining & 0.3063 & 0.1503 & 0.1513 & 0.0867 & 0.0507 & 0.0655 & 0.0401 & 0.0576 \\
\hline 4 & Nonferrous metal mining & 0.3509 & 0.0255 & 0.2031 & 0.0256 & 0.1121 & 0.0254 & 0.0812 & 0.0243 \\
\hline 5 & Nonmetallic mining & 0.0559 & 0.1375 & 0.0181 & 0.0638 & 0.0111 & 0.0648 & 0.0110 & 0.0689 \\
\hline 6 & Other mining & 0.1974 & 0.0334 & 0.0552 & 0.0113 & 0.0429 & 0.0115 & 0.0850 & 0.0164 \\
\hline 7 & Sideline food processing & 0.1010 & 0.0306 & 0.1060 & 0.0497 & 0.0309 & 0.0306 & 0.0298 & 0.0306 \\
\hline 8 & Food manufacturing & 0.0597 & 0.0328 & 0.0321 & 0.0328 & 0.0204 & 0.0322 & 0.2159 & 0.1311 \\
\hline 9 & Beverage manufacturing & 0.1926 & 0.0474 & 0.3729 & 0.0914 & 0.1184 & 0.0498 & 0.1290 & 0.0499 \\
\hline 10 & Tobacco products & 1.0000 & 1.0000 & 1.0000 & 1.0000 & 1.0000 & 1.0000 & 1.0000 & 1.0000 \\
\hline 11 & Textile & 0.0829 & 0.0186 & 0.0699 & 0.0212 & 0.0366 & 0.0184 & 0.0360 & 0.0184 \\
\hline 12 & Textile and garment, shoes, hats manufacturing & 0.3996 & 0.1723 & 0.2140 & 0.1723 & 0.1538 & 0.1723 & 0.1367 & 0.1726 \\
\hline 13 & Leather, fur, feather (velvet) and its products & 0.8041 & 0.0482 & 0.4038 & 0.0483 & 0.1733 & 0.0483 & 0.1628 & 0.0469 \\
\hline 14 & Wood processing and wood, bamboo, rattan, brown, grass products & 0.0895 & 0.1893 & 0.0529 & 0.1877 & 0.0363 & 0.1894 & 0.0286 & 0.1850 \\
\hline 15 & Furniture manufacturing & 0.7134 & 0.2760 & 0.4118 & 0.2773 & 0.1983 & 0.2780 & 0.1902 & 0.2692 \\
\hline 16 & Paper and paper products & 0.0259 & 0.0059 & 0.0475 & 0.0097 & 0.0189 & 0.0057 & 0.0191 & 0.0057 \\
\hline 17 & Printing and recording media & 0.8083 & 0.4015 & 0.4654 & 0.4015 & 0.2140 & 0.4020 & 0.1504 & 0.4035 \\
\hline 18 & Cultural and educational sporting goods manufacturing & 1.0000 & 1.0000 & 1.0000 & 1.0000 & 1.0000 & 1.0000 & 1.0000 & 1.0000 \\
\hline 19 & Oil processing, coking and nuclear fuel processing & 0.0034 & 0.0405 & 0.0019 & 0.0408 & 0.0193 & 0.1924 & 0.0186 & 0.2136 \\
\hline 20 & Chemical raw materials and chemical products manufacturing & 0.0322 & 0.0456 & 0.0252 & 0.0435 & 0.0474 & 0.0739 & 0.0192 & 0.0459 \\
\hline 21 & Pharmaceutical manufacturing & 0.1149 & 0.0415 & 0.0607 & 0.0398 & 0.1160 & 0.0704 & 0.0294 & 0.0400 \\
\hline 22 & Chemical fiber manufacturing & 0.0468 & 0.0176 & 0.1084 & 0.0415 & 0.0155 & 0.0175 & 0.0138 & 0.0177 \\
\hline 23 & Rubber products & 0.0771 & 0.1257 & 0.0434 & 0.1248 & 0.0417 & 0.1249 & 0.0324 & 0.1254 \\
\hline 24 & Plastic products & 0.2279 & 0.3986 & 0.1274 & 0.3944 & 0.1208 & 0.3947 & 0.0975 & 0.3963 \\
\hline 25 & Nonmetallic mineral products & 0.0182 & 0.4051 & 0.0186 & 0.4401 & 0.0159 & 0.3825 & 0.0163 & 0.4253 \\
\hline 26 & Ferrous metal smelting and rolling processing & 0.0316 & 0.2242 & 0.0147 & 0.1324 & 0.0062 & 0.0744 & 0.0076 & 0.0880 \\
\hline 27 & Nonferrous metal smelting and rolling processing & 0.0274 & 0.1355 & 0.0151 & 0.1318 & 0.0104 & 0.1332 & 0.0105 & 0.1599 \\
\hline 28 & Metal products & 0.4010 & 0.0918 & 0.1874 & 0.0940 & 0.1000 & 0.0916 & 0.1031 & 0.0919 \\
\hline 29 & General equipment manufacturing & 0.4783 & 0.3583 & 0.4099 & 0.3582 & 0.2655 & 0.3583 & 0.2620 & 0.3599 \\
\hline 30 & Special equipment manufacturing & 0.2075 & 0.2944 & 0.1545 & 0.2904 & 0.1686 & 0.2894 & 0.1612 & 0.2975 \\
\hline 31 & Transportation equipment manufacturing & 0.3265 & 0.2436 & 0.2604 & 0.2436 & 0.2653 & 0.2436 & 0.2649 & 0.2506 \\
\hline 32 & Electrical machinery and equipment manufacturing & 1.0000 & 1.0000 & 1.0000 & 1.0000 & 1.0000 & 1.0000 & 1.0000 & 1.0000 \\
\hline 33 & $\begin{array}{c}\text { Communications equipment, computers and other electronic } \\
\text { equipment manufacturing }\end{array}$ & 1.0000 & 1.0000 & 1.0000 & 1.0000 & 1.0000 & 1.0000 & 1.0000 & 1.0000 \\
\hline 34 & Instrumentation and culture, office machinery manufacturing & 1.0000 & 1.0000 & 1.0000 & 1.0000 & 1.0000 & 1.0000 & 1.0000 & 1.0000 \\
\hline 35 & Handicrafts and other manufacturing & 0.0752 & 0.3283 & 1.0000 & 1.0000 & 1.0000 & 1.0000 & 1.0000 & 1.0000 \\
\hline 36 & Waste resources and waste materials recycling & 0.4504 & 0.1381 & 0.2425 & 0.1381 & 0.0552 & 0.1381 & 0.0445 & 0.1361 \\
\hline 37 & Electricity, heat production and supply & 0.0042 & 0.1713 & 0.0049 & 0.1997 & 0.0028 & 0.1246 & 0.0061 & 0.2009 \\
\hline 38 & Gas production and supply & 0.0423 & 0.3446 & 0.0072 & 0.1717 & 0.0171 & 0.2539 & 0.0077 & 0.1537 \\
\hline 39 & Water production and supply & 0.3160 & 0.0115 & 0.1253 & 0.0115 & 0.1464 & 0.0117 & 0.1456 & 0.0113 \\
\hline
\end{tabular}


The TFE of coal input and wastewater control of different industries from 2003 to 2014 are also shown in Figure 3 below.
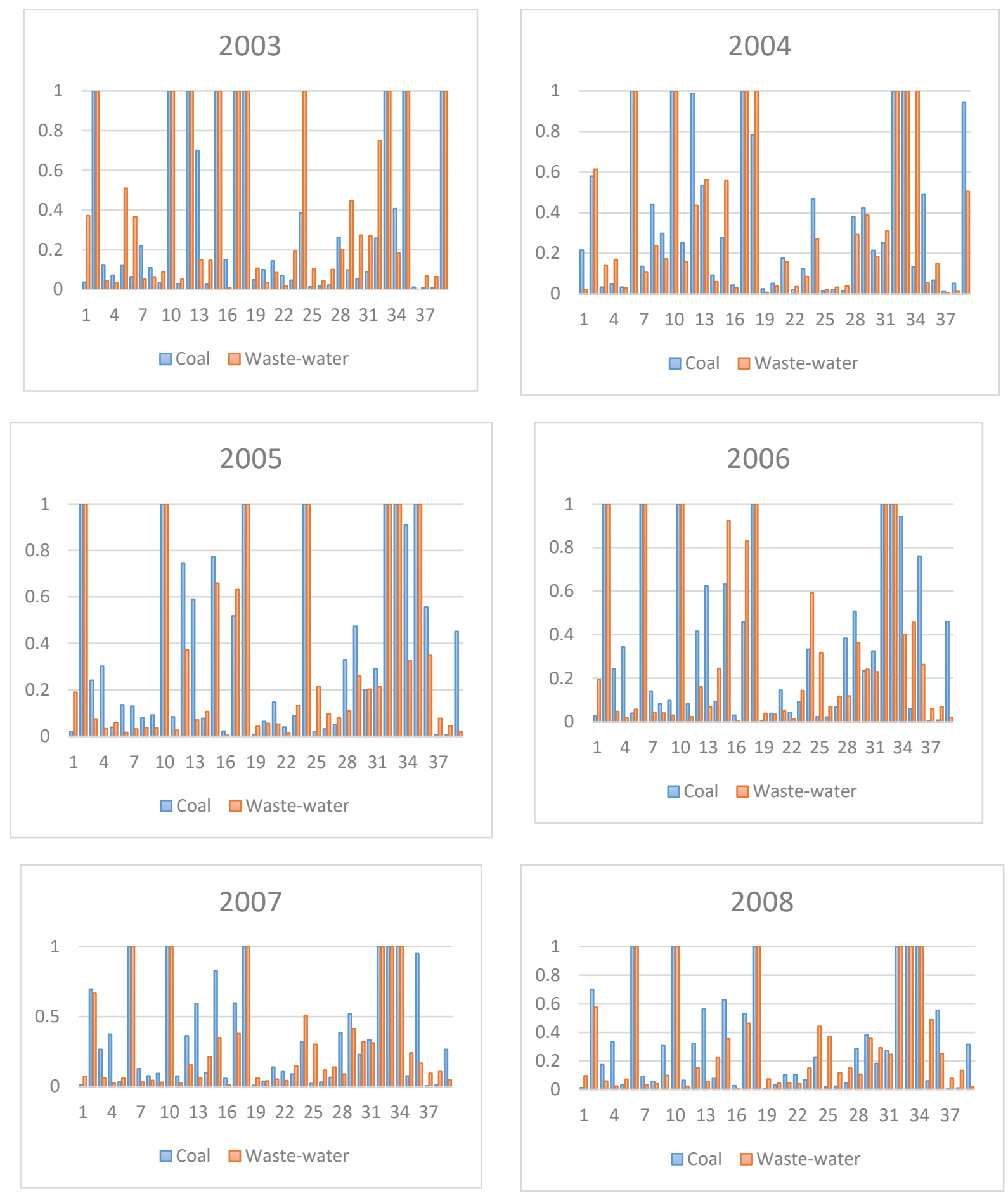

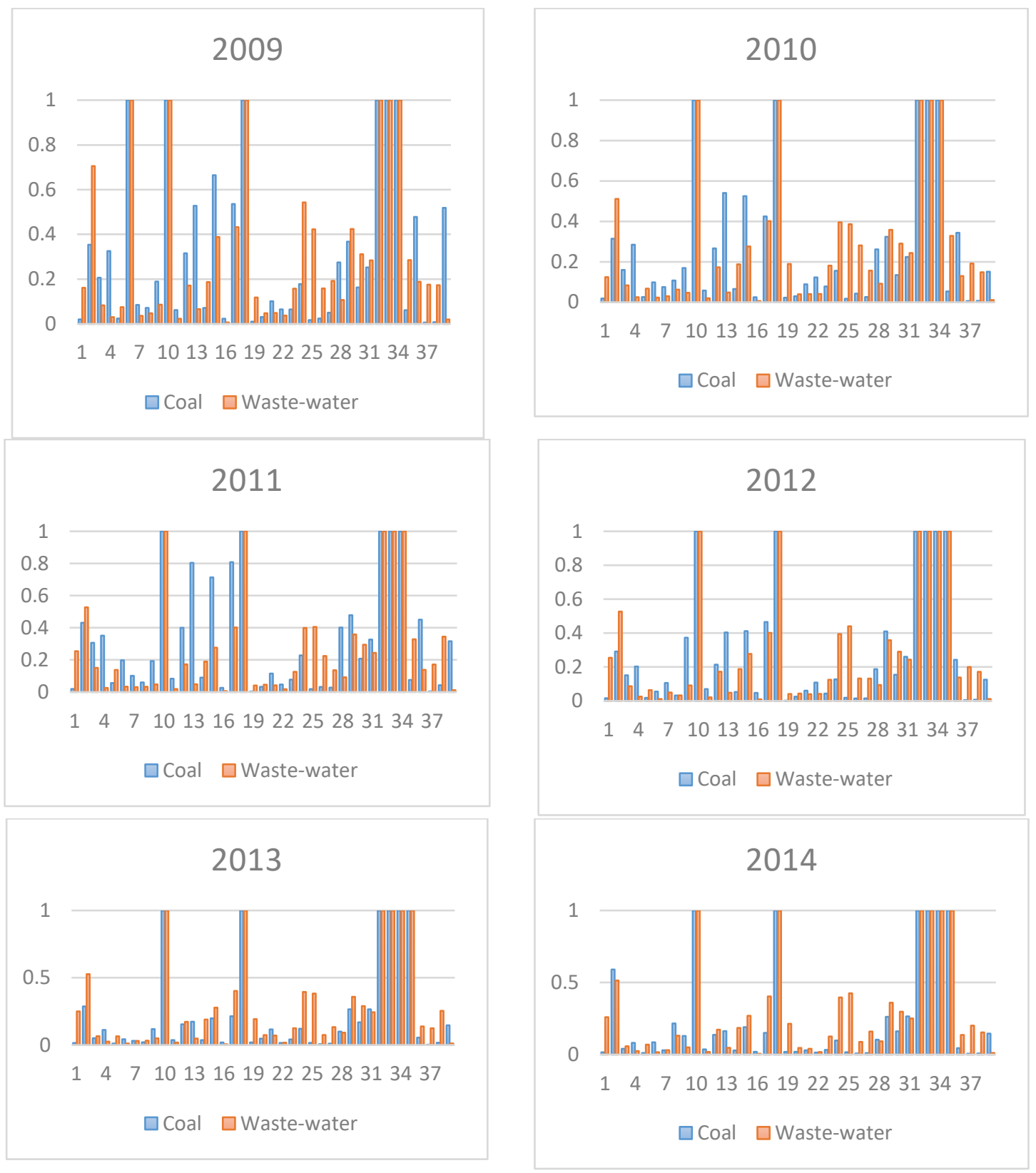

Figure 3. TFE of coal input and wastewater control in different industries in China, 2003-2014 (the serial numbers from 1 to 39 in Figure 3 correspond to the industries in Table 1).

\section{Discussion}

Based on results in Tables 2-4 and Figure 3, the main characteristics of coal input TFE and wastewater control TFE of the various industries in China from 2003 to 2014 can be summarized and analyzed as below:

\section{(1) The polarization of TFE results}

In terms of the TFE in coal input and wastewater control of various industries in China, the calculation results showed huge gap between different industries. Take the example of 2014-there were six industries (No. 10, 18, 32, 33, 34 and 35) that reached the optimal Dual Target Variable efficiency of 1, including the tobacco products industry (No. 10); the efficiency of the oil and gas extraction industry (No. 2) exceeded 0.5 , while the rest 32 industries (or $82.05 \%$ of the total industries 
within our study scope) only ended up with an efficiency level below 0.5. Among those 32 industries, there were 11 industries (No. 3, 4, 5, 6, 7, 11, 16, 20, 21, 22 and 26) whose Dual Target Variable efficiency was lower than 0.1 , accounting for $28.21 \%$ of the industries within our study scope. Over the entire study period of 2003-2014, only national monopoly industries (such as the No. 10 tobacco industry) and high and new tech industries (such as the No. 33 communications equipment, computers and other electronic equipment manufacturing industry) were able to maintain the optimal efficiency level of 1 , while the remaining industries experienced different levels of fluctuation in terms of Dual Target Variable efficiency.

\section{Good TFE results of manufacturing industries}

Manufacturing industries showed outstanding performance in terms of TFE throughout the study period. The coal input TFE and wastewater control TFE of the communications equipment, computers and other electronic equipment manufacturing industry (No. 33) maintained optimal level throughout the study period. The electrical machinery and equipment manufacturing industry (No. 32) achieved a high efficiency level during the study period, except in 2003. The instrumentation and culture, office machinery manufacturing industry (No. 34) experienced some fluctuation during 2003-2006 in terms of TFE, but they all reached optimal efficiency in the following years. The cultural and educational sporting goods manufacturing industry (No. 18) maintained optimal efficiency throughout the study period, except for the coal input efficiency in 2004. Despite some fluctuation, the handicrafts and other manufacturing industry (No. 35) also achieved optimal efficiency in 2003, 2005, and 2012-2014. It is worth noticing that since 2003, China has initiated the second round of development planning for the manufacturing industry since the reform-and-opening-up, which focuses on the fields of equipment manufacturing, shipbuilding, high-speed train, nuclear power and new energy and has achieved world-leading progress. By 2014, which is also the end of our study period, the "Made in China 2025" Strategy, which aims to comprehensively improve the quality of Chinese manufacturing industry by 2045 has also taken shape [37]. The time between 2003 and 2014 was the "Golden Age" of the Chinese manufacturing industry. According to the statistics of the United Nations Industrial Development Organization, in 2014, the net export of the Chinese manufacturing industry ranked first worldwide, and among the 22 industry categories defined by the International Standard Industrial Classification, China achieved top rank in seven industry categories with more than 220 industrial goods' production ranking first worldwide, including steel, cement and automobile [38].

\section{Poor TFE performance of natural resource industries}

The natural resource industries suffered poor TFE performance. The oil and gas extraction industry (No. 2) reached optimal efficiency of 1 in 2003, 2005 and 2006, but experienced huge fluctuation in the other years. Its efficiency level at the end of the period (2014) was only $59.03 \%$ and $51.42 \%$ at its beginning in 2003. The ferrous metal smelting and rolling processing industry (No. 26) and the nonferrous metal smelting and rolling processing industry (No. 27) shared a similar trend in TFE. Their coal input TFE reached top levels during 2005-2011, and a sharp drop afterwards. In contrast, their wastewater control TFE did not show similar fluctuation, as its coal input TFE during the study period. One possible reason is this: As the direct oversight and management department of these resource industries, the National Development and Reform Commission (NDRC) of China abolished the old industry standards and started to revise 16 oil and gas industry standards, 61 ferrous metal metallurgy industry standards, and 46 nonferrous metal industry standards in 2005 [39]. This industry standard amendment enhanced the input-output efficiency of these industries to some extent. In addition, since the mid-2005, the supply and demand of the coal industry reached a balance and the coal price slowed down its pace of increase. Therefore, the coal input efficiency of these industries has shown clear improvement since 2005. However, in terms of sustainability, improvement in coal input TFE is driven by policy factors cannot continue in the long term. 
(2) The effects of "Chinese characteristics" policies

I. Effect on the electricity, heat production and supply industry (No. 37)

As the biggest consumer of coal resources throughout the study period, the electricity, heat production and supply industry [25] has maintained a comparatively low TFE in coal input and wastewater control. Except for 2004, its TFE in coal input was below 0.01 throughout the study period; its TFE in industrial wastewater control was below 0.1 from 2003 to 2008, which improved a little during 2009-2014, but only achieved 0.2009 as the highest level. The low efficiency of the power industry has its own historical reasons, and reflects the inherent characteristics of the Chinese energy system, where government interference overrules market mechanism.

The electric supply industry reform in China began in the early 1990s. At that time, the electric supply industry established seven nationwide power enterprises, including Huaneng, five regional power groups, and Gezhou Dam Hydropower Corporation. Although these enterprises represented state-owned capital, and their operation and management were based on administrative orders, until the market reform focusing on "Separating Plant and Grid and Electric Power Bidding" in 2002, the electric supply industry had already reached a high level of marketization compared with other industries in the energy sector [40]. However, under the unique energy management system of China, the pricing of energy products is determined by the National Development and Reform Commission (NDRC) [41]. Therefore, although the market reform within the electric supply industry itself had made great achievement, despite the continuous increase in coal price since 2001, the price of electric power to the end user failed to rise. Furthermore, to make it worse for the TFE of this industry, the government did not allow the market mechanism to adjust electric power price automatically given coal price increase, but issued a price adjustment policy in 2004-the "Coal-Electricity Price Linkage" policy - that had a profound and lasting impact on the electric supply and coal industries. According to this "price linkage" policy, with a review cycle of no less than six months, the cost of electric power will only be adjusted when the average coal price in that cycle has changed more than $5 \%$ compared to the last cycle [42]. In 2012, the State Council of China furtherly issued a policy that the on-grid price will only be adjusted if the coal price has changed more than $5 \%$ compared with last year [43]. Within the study period, the total upward price adjustment of electric power due to coal price increase was 11.92 cents/KWH, while the total downward price adjustment due to coal price decrease was 7.44 cents/KWH under this "price linkage" policy, which means that the actual movement of the electric power price within this period was merely 4.48 cents/KWH [44].

This "price linkage" policy had brought huge negative impact on the Dual Target Variable TFE of the electric power industry: (1) As a price adjustment mechanism regulated by the government, the "price linkage" policy cannot absorb the impact of coal price change on electric power enterprises timely as in the free market. (2) In reality, this policy cannot guarantee simultaneous movement of coal price and the price of electric power. According to this policy, there will be at least six months lag between coal price change and electric power price adjustment (since 2012, the time lag has increased to 12 months). Moreover, if the fluctuation of coal price did not reach $5 \%$, then the price of electric power would not be possible. Such stubborn system deficiency had severely impaired the coal input TFE of the electric power industry in China. (3) This policy neglected the variety of electric power enterprises in China, i.e., their difference in type, region and coal cost. The fixed threshold of $5 \%$ coal price change had caused inflexibility and had a negative influence on the coal input TFE of different electric power enterprises. (4) This "price linkage" mechanism did not take into account the output side, especially undesirable outputs (industrial wastewater emission). Therefore, from the perspective of policy orientation, this policy overemphasized the input and cost factors, but neglected wastewater control and environmental concerns. Although the more strict environmental protection policies and regulations after 2009 [45-49] improved wastewater control TFE of the electric power industry over the entire study period, we have to admit that this "price linkage" mechanism in fact did not have a positive influence on the wastewater control TFE of this industry. 
II. Effect on the coal mining and washing industry (No. 1)

As a direct coal production and major coal consumption industry, the coal mining and washing industry (No. 1) has maintained a low coal input TFE. Except for 2004 in which it reached a TFE of 0.2159 , its coal input TFE had always been below 0.04 throughout the study period. The "price linkage" policy that started in 2004 clearly benefits the coal input TFE of coal production companies. First, this mechanism squeezed the abnormal profits between coal production and sales, which would help guarantee the completion of contracts and therefore improve coal input efficiency. Second, this policy generated greater anticipation on the future return to coal production and therefore further motivated the competitiveness of the coal production industry. However, as mentioned above, this planned economy mechanism cannot achieve the same price adjustment result as under market mechanism. The coal input TFE did increase in 2004, but the improvement was quite limited and the TFE dropped quickly after 2004, which proved that although the planned economy mechanism may have some positive effect, it would be very limited and unsustainable.

On the other hand, the wastewater control efficiency of the coal mining and washing industry was also quite low throughout the study period, which reached its highest level of 0.3717 in 2003, while remained a declining trend in the following years. Its wastewater control efficiency improved a little in 2009 , but still failed to exceed 0.3 . The main reason behind may be the fact that currently there is still $13.37 \%$ of the coal-washing wastewater that escapes from the closed cycle [50]. With increasing coal input, more coal-washing wastewater escapes from the closed cycle and is emitted into the environment, which will not only hurt the wastewater control TFE of the coal mining and washing industry, but also pose a great threat to the environment.

\section{Conclusions}

Based on our previous studies [51-54] and improved DEA Model specialized for Dual Target Variable TFE calculation with the MATLAB algorithm developed by us here, this paper conducted both theoretical and empirical study on the Dual Target Variable TFE of both coal input and wastewater control of various industry sectors in China from 2003 to 2014 . We found that 32 industries $(82.05 \%$ of the total industries within our study scope) ended up with an efficiency level below 0.5. Among those industries, there were 12 industries whose Dual Target Variable efficiency was lower than 0.1. Moreover, only the national monopoly industries and high and new tech industries maintained the optimal efficiency level of 1 during the study period of 2003-2014, while the rest experienced different levels of fluctuation in Dual Target Variable TFE, especially in the electric and thermal power industry, and the coal mining and washing industry.

Overall, the coal input and wastewater control TFE of these 39 industries was quite low, which has to some extent become a major obstacle for the sustainable development of the Chinese industry. Therefore, we propose the following policy recommendations:

(1) Improve technologies and innovation skills in the field of coal utilization and wastewater treatment. Currently, coal mining technology and equipment in China's major coal production areas are not very advanced, resulting in a huge gap in terms of coal utilization and wastewater treatment capabilities, compared with the international standard. Therefore, advanced mining, smelting and cleaning technologies in the exploitation and industrial production stage of coal resources should be actively developed and adopted in order to minimize the environment impact of wastewater emission from coal production with the help of modern technologies. At the same time, the R\&D investment in the field of coal utilization and wastewater treatment in the industrial production process should be increased.

(2) The government should also learn from the lessons of the "coal-electric power price linkage" policy and introduce more market mechanisms. For example, it could develop and implement a compensation mechanism on natural resource utilization and environment pollution, and 
minimize the external cost and external benefit with the help of market mechanism and market economy.

Author Contributions: L.L. organized the whole research, performed calculations, designed the methodology and MATLAB program, and provided the final figures. As the correspondence author, W.Y. collected original data, and analyzed the data and background materials. Both authors carefully read and approved the final manuscript.

Funding: This research was funded by the National Natural Science Foundation of China, grant number [11501418], the Shanghai Sailing Program grant number [15YF1412500], the Humanities and Social Sciences Research Fund of University of Shanghai for Science and Technology, and the Shanghai Municipal People's Government Decision-making Consultation Research Project.

Acknowledgments: The first author was financially supported by the National Natural Science Foundation of China [grant number 11501418] and the Shanghai Sailing Program [grant number 15YF1412500]. The second author was financially supported by the Humanities and Social Sciences Research Fund of University of Shanghai for Science and Technology, and the Shanghai Municipal People's Government Decision-making Consultation Research Project. We gratefully acknowledge the above financial supports.

Conflicts of Interest: The authors declare no conflict of interest.

\section{Appendix A}

We designed the following MATLAB algorithm to calculate the TFE of both coal input and industrial wastewater control.

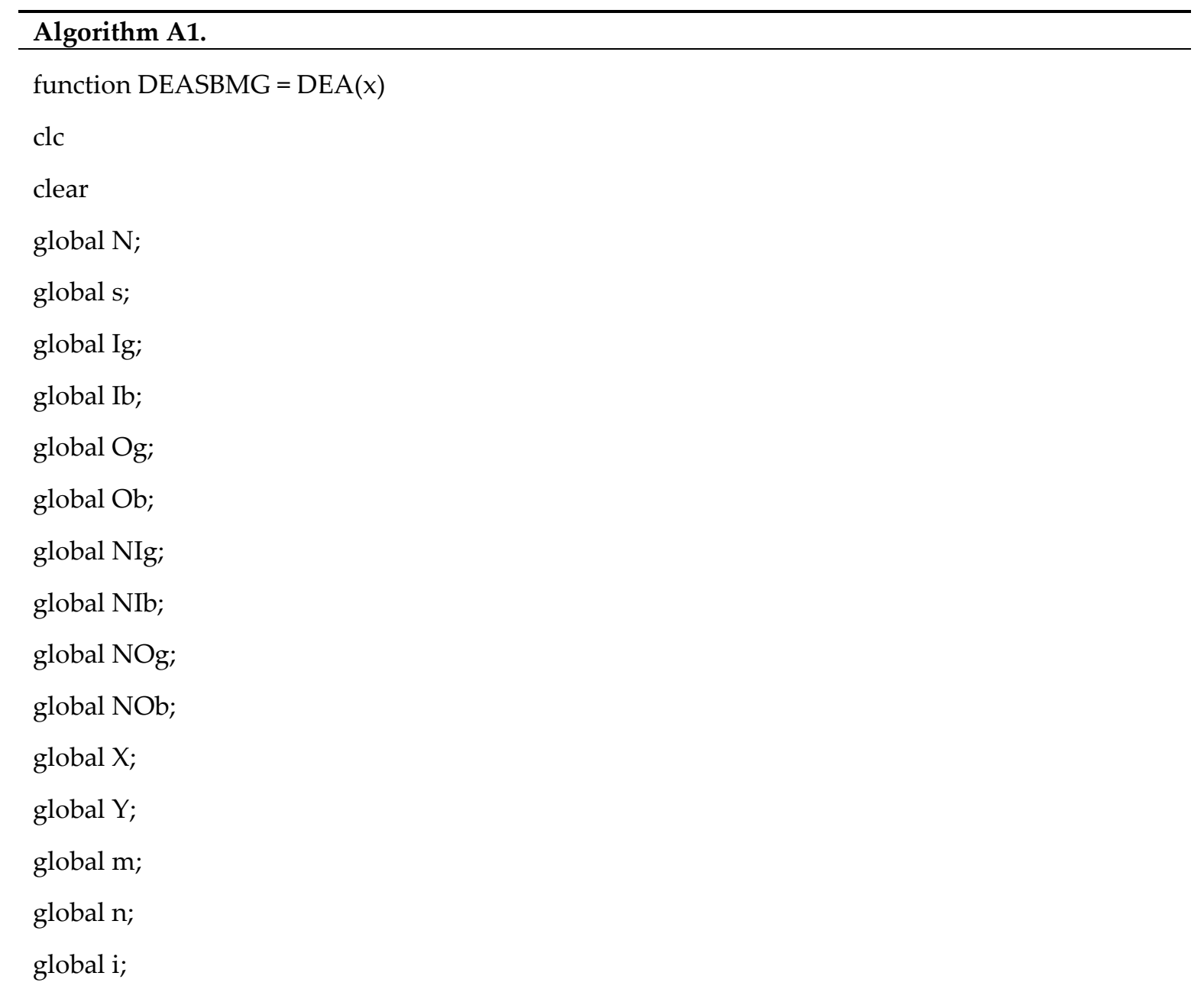


$\mathrm{Xg}=[] ;$

$\mathrm{Xb}=[]$;

$\mathrm{Yg}=[] ;$

$\mathrm{Yb}=[]$;

Var=[];

$[\mathrm{s}, \mathrm{N}]=\operatorname{size}($ Var);

$\mathrm{r} 0=$ zeros $(\mathrm{s}, 1)$;

$\mathrm{R}=\mathrm{zeros}(\mathrm{s}, \mathrm{s})$;

fval=zeros $(\mathrm{s}, \mathrm{N})$;

Theta=zeros(s,N);

ThetaIgI=zeros(s,N);

ThetaIgII=zeros $(\mathrm{s}, \mathrm{N})$;

ThetaIgIII=zeros $(\mathrm{s}, \mathrm{N})$;

ThetaOg=zeros $(\mathrm{s}, \mathrm{N})$;

ThetaObI=zeros $(\mathrm{s}, \mathrm{N})$;

ThetaObII=zeros $(\mathrm{s}, \mathrm{N})$;

ThetaObIII=zeros $(\mathrm{s}, \mathrm{N})$;

for $k=1: N$

$\mathrm{Ig}=\mathrm{Xg}^{\prime}$;

$\mathrm{Ib}=\mathrm{Xb}^{\prime}$;

$\mathrm{Og}=\operatorname{Var}(:, \mathrm{k})^{\prime}$;

$\mathrm{Ob}=\mathrm{Yb}^{\prime}$;

$\mathrm{X}=[\mathrm{Ig} ; \mathrm{Ob}]$;

$\mathrm{Y}=[\mathrm{Ib} ; \mathrm{Og}]$;

[NIg,s]=size(Ig);

$[\mathrm{NIb}, \mathrm{s}]=\operatorname{size}(\mathrm{Ib})$;

$[\mathrm{NOg}, \mathrm{s}]=$ size $(\mathrm{Og})$;

$[\mathrm{NOb}, \mathrm{s}]=\operatorname{size}(\mathrm{Ob})$;

$[\mathrm{m}, \mathrm{s}]=\operatorname{size}(\mathrm{X})$;

$[\mathrm{n}, \mathrm{s}]=\operatorname{size}(\mathrm{Y})$;

$\mathrm{A}=[\mathrm{X} ;-\mathrm{Y}]$;

for $i=1$ :s 


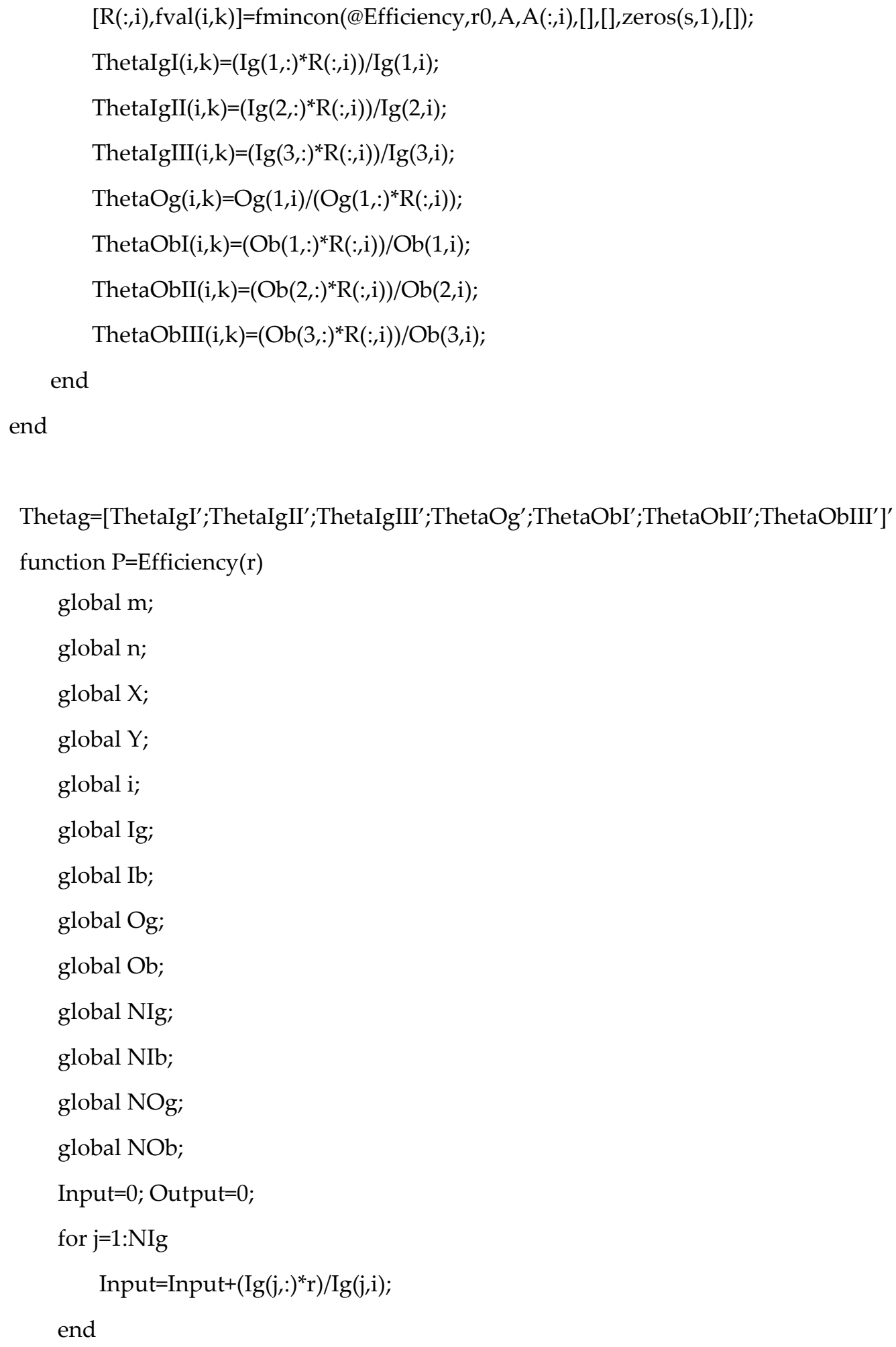


for $\mathrm{j}=1: \mathrm{NIb}$

Input=Input+Ib(j,i)/(Ib(j,: $\left.)^{*} r\right) ;$

end

for $\mathrm{j}=1 \mathrm{NOg}$

Output=Output $+\left(\mathrm{Og}(\mathrm{j},:)^{*} \mathrm{r}\right) / \mathrm{Og}(\mathrm{j}, \mathrm{i})$;

end

for $\mathrm{j}=1: \mathrm{NOb}$

Output=Output+Ob(j,i)/(Ob(j,::**);

end

$\mathrm{P}=\left((\mathrm{NOg}+\mathrm{NOb})^{*} \mathrm{Input}\right) /\left((\mathrm{NIg}+\mathrm{NIb})^{*} \mathrm{Output}\right) ;$

\section{References}

1. National Bureau of Statistics of China. China Statistical Yearbook, 1979-2016; China Statistic Press: Beijing, China, 2016.

2. National Bureau of Statistics of China. China Statistical Yearbook, 2001-2016; China Statistic Press: Beijing, China, 2016.

3. Zhang, D.; Wang, J.; Lin, Y.; Si, Y.; Huang, C.; Yang, J.; Huang, B.; Li, W. Present situation and future prospect of renewable energy in China. Renew. Sustain. Energy Rev. 2017, 76, 865-871. [CrossRef]

4. Peng, H.; Liu, Y. How government subsidies promote the growth of entrepreneurial companies in clean energy industry: An empirical study in China. J. Clean. Prod. 2018, 188, 508-520. [CrossRef]

5. Wang, B.; Wang, Q.; Wei, Y.-M.; Li, Z.-P. Role of renewable energy in China's energy security and climate change mitigation: An index decomposition analysis. Renew. Sustain. Energy Rev. 2018, 90, 187-194. [CrossRef]

6. Xie, W.; Sheng, P.; Guo, X. Coal, oil, or clean energy: Which contributes most to the low energy efficiency in China? Util. Policy 2015, 35, 67-71. [CrossRef]

7. Yang, X.J.; Hu, H.; Tan, T.; Li, J. China's renewable energy goals by 2050. Environ. Dev. 2016, 20, 83-90. [CrossRef]

8. Buzzi, D.C.; Viegas, L.S.; Rodrigues, M.A.S.; Bernardes, A.M.; Tenório, J.A.S. Water recovery from acid mine drainage by electrodialysis. Miner. Eng. 2013, 40, 82-89. [CrossRef]

9. Bai, H.; Kang, Y.; Quan, H.; Han, Y.; Sun, J.; Feng, Y. Treatment of acid mine drainage by sulfate reducing bacteria with iron in bench scale runs. Bioresour. Technol. 2013, 128, 818-822. [CrossRef] [PubMed]

10. Simate, G.S.; Ndlovu, S. Acid mine drainage: Challenges and opportunities. J. Environ. Chem. Eng. 2014, 2, 1785-1803. [CrossRef]

11. Ma, Q.; Qu, Y.Y.; Zhang, X.W.; Shen, W.L.; Liu, Z.Y.; Wang, J.W.; Zhang, Z.-J.; Zhou, J.-T. Identification of the microbial community composition and structure of coal-mine wastewater treatment plants. Microbiol. Res. 2015, 175, 1-5. [CrossRef] [PubMed]

12. Chang, S.; Zhuo, J.; Meng, S.; Qin, S.; Yao, Q. Clean coal technologies in China: Current status and future perspectives. Engineering 2016, 2, 447-459. [CrossRef]

13. Wang, Y.; Song, Y.; Zhou, H.; Zhi, K.; Teng, Y.; He, R.; Tian, R.; Liu, Q. Comparative study on structural properties and combustion kinetics of Chinese Shenhua long flame raw coal and its pyrolytic char. Environ. Prog. Sustain. Energy 2017, 36, 766-774. [CrossRef]

14. Gai, H.; Song, H.; Xiao, M.; Feng, Y.; Wu, Y.; Zhou, H.; Chen, B. Conceptual design of a modified phenol and ammonia recovery process for the treatment of coal gasification wastewater. Chem. Eng. J. 2016, 304, 621-628. [CrossRef]

15. Qian, Y.; Yang, S.Y.; Ma, D.; Cui, P. Research advances in treatment of coal gasification wastewater with high phenol and ammonia. Chem. Ind. Eng. Prog. 2016, 35, 1884-1893. 
16. Geng, Z.Q.; Dong, J.G.; Han, Y.M.; Zhu, Q.X. Energy and environment efficiency analysis based on an improved environment DEA cross-model: Case study of complex chemical processes. Appl. Energy 2017, 205, 465-476. [CrossRef]

17. U.S. Environmental Protection Agency. Watershed-Based National Pollutant Discharge Elimination System (NPDES) Permitting Technical Guidance; U.S. Environmental Protection Agency: Washington, DC, USA, 2007.

18. Selman, M. Water quality trading programs: An international overview. WRI Issue Br. Water Qual. Trading 2009, 1, 1-16.

19. Office of Wastewater Management U.S. Environmental Protection Agency. Emerging Technologies for Wastewater Treatment and In-Plant Wet Weather Management; Office of Wastewater Management U.S. Environmental Protection Agency: Washington, DC, USA, 2013.

20. McCarty, P.L.; Bae, J.; Kim, J. Domestic wastewater treatment as a net energy producer-Can this be achieved? Environ. Sci. Technol. 2011, 45, 7100-7106. [CrossRef] [PubMed]

21. Al-Isawi, R.H.K.; Scholz, M.; Al-Faraj, F.A.M. Assessment of diesel-contaminated domestic wastewater treated by constructed wetlands for irrigation of chillies grown in a greenhouse. Environ. Sci. Pollut. Res. 2016, 23, 25003-25023. [CrossRef] [PubMed]

22. Fu, G.; Butler, D.; Khu, S.T. Multiple objective optimal control of integrated urban wastewater systems. Environ. Model. Softw. 2008, 23, 225-234. [CrossRef]

23. Schütze, M.; Butler, D.; Beck, B.M. Modelling, Simulation and Control of Urban Wastewater Systems; Springer: London, UK, 2012.

24. Bach, P.M.; Rauch, W.; Mikkelsen, P.S.; McCarthy, D.T.; Deletic, A. A critical review of integrated urban water modelling-Urban drainage and beyond. Environ. Model. Softw. 2014, 54, 88-107. [CrossRef]

25. National Bureau of Statistics of China. China Statistical Yearbook, 2004-2015; China Statistic Press: Beijing, China, 2015.

26. Zhang, J.; Wu, G.; Zhang, J. The estimation of China's provincial capital stock: 1952-2000. Econ. Res. J. 2004, $10,35-44$.

27. Department of Energy Statistics, National Bureau of Statistics of China. China Energy Statistical Yearbook, 2004-2015; China Statistic Press: Beijing, China, 2015.

28. Reinhard, S.; Lovell, C.A.K.; Thijssen, G.J. Environmental efficiency with multiple environmentally detrimental variables; estimated with SFA and DEA. Eur. J. Oper. Res. 2000, 121, 287-303. [CrossRef]

29. Färe, R.; Grosskopf, S. Directional distance functions and slacks-based measures of efficiency. Eur. J. Oper. Res. 2010, 200, 320-322. [CrossRef]

30. Chen, S.; Zhang, S. Large-scale land use for construction and energy consumption after the new millennium with their impact on total-factor efficiency change in China's regional economy. Energies 2014, 7, 1986-2003. [CrossRef]

31. Ma, Y.; Jiang, C.; Hou, Z.; Wang, C. The formulation of the optimal strategies for the electricity producers based on the particle swarm optimization algorithm. IEEE Trans. Power Syst. 2006, 21, 1663-1671. [CrossRef]

32. Tolson, B.A.; Shoemaker, C.A. Dynamically dimensioned search algorithm for computationally efficient watershed model calibration. Water Resour. Res. 2007, 43. [CrossRef]

33. Ebbesen, S.; Kiwitz, P.; Guzzella, L. A generic particle swarm optimization Matlab function. In Proceedings of the 2012 American Control Conference (ACC), Montreal, QC, Canada, 27-29 June 2012; pp. 1519-1524.

34. Cheng, S.-L.; Hwang, C. Optimal approximation of linear systems by a differential evolution algorithm. IEEE Trans. Syst. Man Cybern. A Syst. Hum. 2001, 31, 698-707. [CrossRef]

35. Cook, W.D.; Liang, L.; Zhu, J. Measuring performance of two-stage network structures by DEA: A review and future perspective. Omega 2010, 38, 423-430. [CrossRef]

36. Lim, S.; Zhu, J. Integrated data envelopment analysis: Global vs. local optimum. Eur. J. Oper. Res. 2013, 229, 276-278. [CrossRef]

37. The State Council of the People's Republic of China. Made in China 2025; People's Publishing House: Beijing, China, 2015.

38. United Nations Industrial Development Organization. UNIDO Annual Report 2014; United Nations Industrial Development Organization: Vienna, Austria, 2015.

39. National Development and Reform Commission Abolition of "Refractory Bauxite Classification and Technical Conditions" and Other 61 Black Metallurgical Industry Standards. Available online: http: / / www.mofcom.gov.cn/aarticle/b/g/200508/20050800308551.html (accessed on 15 November 2017). 
40. Yang, W.X. China's Energy Security in the Context of International Energy (Doctoral Dissertation); Shanghai Academy of Social Sciences: Shanghai, China, 2006.

41. National Development and Reform Commission Main Functions of the NDRC. Available online: http: / / en.ndrc.gov.cn/mfndrc/ (accessed on 15 November 2017).

42. National Development and Reform Commission the Notice of Issuing the Statement on the Establishment of Coal-Electricity Price Linkage Mechanism by NDRC. Available online: http:/ /bgt.ndrc.gov.cn/zcfb/200807/ t20080710_499686.html (accessed on 15 November 2017).

43. The General Office of the State Council of the People's Republic of China Guiding opinions of the General Office of the State Council on Deepening Coal Market Reform. Available online: http:/ /www.gov.cn/zwgk/ 2012-12/25/content_2298187.htm (accessed on 15 November 2017).

44. Economic Forecast Department of State Information Center of China Impact Analysis of the Cut of Coal On-Grid Price. Available online: http://www.sic.gov.cn/News/466/7188.htm (accessed on 15 November 2017).

45. Standing Committee of the National People's Congress of the People's Republic of China. The People's Republic of China Renewable Energy Law Amendment; The Law Press: Beijing, China, 2009.

46. Standing Committee of the National People's Congress of the People's Republic of China. Cleaner Production Promotion Law of the People's Republic of China (2012 Amendment); China Democracy and Legal system Publishing House: Beijing, China, 2012.

47. Standing Committee of the National People's Congress of the People's Republic of China. Law of the People's Republic of China on the Prevention and Control of Environmental Pollution by Solid Wastes (Amendment); China Democracy and Legal system Publishing House: Beijing, China, 2013.

48. Standing Committee of the National People's Congress of the People's Republic of China. Environmental Protection Law of the People's Republic of China (2014 Revision); China Legal Publishing House: Beijing, China, 2014.

49. Standing Committee of the National People's Congress of the People's Republic of China. Marine Environmental Protection Law of the People's Republic of China (2017 Amendment); China Legal Publishing House: Beijing, China, 2017.

50. Li, Y.F. Study on Treatment and Reuse Technology of Concenrtated Wastewater of Coal Washing. Ph.D. Thesis, Northeastern University, Boston, MA, USA, 2005.

51. Yang, W.X.; Li, L.G. Efficiency evaluation of industrial waste gas control in China: A study based on data envelopment analysis (DEA) model. J. Clean. Prod. 2018, 179, 1-11. [CrossRef]

52. Yang, W.X.; Li, L.G. Efficiency Evaluation and Policy Analysis of Industrial Wastewater Control in China. Energies 2017, 10, 1201. [CrossRef]

53. Yang, W.X.; Li, L.G. Analysis of total factor efficiency of water resource and energy in China: A study based on DEA-SBM model. Sustainability 2017, 9, 1316. [CrossRef]

54. Yang, W.X.; Li, L.G. Energy efficiency, ownership structure, and sustainable development: Evidence from China. Sustainability 2017, 9, 912. [CrossRef]

(c) 2018 by the authors. Licensee MDPI, Basel, Switzerland. This article is an open access article distributed under the terms and conditions of the Creative Commons Attribution (CC BY) license (http:/ / creativecommons.org/licenses/by/4.0/). 